\title{
VARIATIONAL PROPERTY OF CUSP FORMS
}

\author{
BY \\ HARVEY COHN(1)
}

1. Introduction. In an earlier paper [1] a geometric construction was given for defining a certain Eisenstein series of dimension (always) -4, namely

$$
E(z)=\sum(q z-p)^{-4},
$$

$\operatorname{Im} z>0$

summed over all reduced rational fractions $p / q$ (including $1 / 0$ ). The construction involved perturbations. A modification of the construction could be used to define Eisenstein series corresponding to subgroups of the modular group. Further investigation, however, reveals that perturbation constructions will yield only Eisenstein series, (meaning that the cusp forms are not so constructible). Although the last result appears negative it leads to another result whose statement constitutes the main theorem (see $\$ 2$ below). The statement is independent of perturbation theory altogether and it enables us to detect the presence of a cusp form by the so-called "period-polynomials" of a modular form. It also is possible to construct a formalism which is in itself of interest. In fact a comparable formalism has been discovered by another method by Bol (see [5]), but apparently has never been applied to the present purpose.

The method of variation of boundary has been used in the past not only for minimal problems but also for establishing identities among modules of Riemann surfaces (see $[7$, p. 316]). According to a private communication, M. Schiffer has, in unpublished work, used the method of interior variations (see $[7$, p. 283]) to define and establish the dimension of theta-functions with poles defined on the covering surface of a punctured plane. The present work by contrast might be said, roughly speaking, to use perturbations around the boundary (or cusp) points of the covering surface, leading to Eisenstein series (without poles), or, as is even more important, leading only to these Eisenstein series. As is well known, the Eisenstein series have rather elementary Fourier coefficients [2] which depend, for instance, on divisor functions and it is therefore of value to be able to say that a modular function is linearly expressible in terms of Eisenstein series alone.

2. Main theorem. We consider a congruence sugbroup $\mathcal{G}$ of linear fractional transformations, $T, S, \cdots$,

Presented to the Society, September 3, 1955; received by the editors July 25, 1955.

(1) Research supported in part by contract Nonr-225(11) with the Office of Naval Research. 


$$
z \rightarrow T z=\frac{a z+b}{c z+d}, \quad T=\left(\begin{array}{ll}
a & b \\
c & d
\end{array}\right) .
$$

(Here $a, b, c, d$ are integers for which $a d-b c=1$.) Let $k(\geqq 1)$ denote the number of inequivalent cusp points or the number of rational points $\left(r_{1}, r_{2}, \cdots\right.$, $r_{k}$ ) such that $r_{i} \neq T r_{j}(i \neq j)$. We define $k$ distinct Eisenstein series

$$
E\left(z ; r_{t}\right)=\sum_{p / q \sim r_{t}}(q z-p)^{-4}, \quad t=1,2, \cdots, k,
$$

where the summation refers to all reduced fractions $p / q$ (including possibly $1 / 0)$, in the set $\operatorname{Tr}_{t}$, where $T$ varies over $G$. We can easily verify the fact that $\phi(z)=E\left(z ; r_{t}\right)$ satisfies the following conditions describing a modular form of dimension -4 :

$$
\begin{aligned}
& (c z+d)^{-4} \phi(T z)=\phi(z) \\
& \phi(z)=O(q z-p)^{-4} \text { as } z \rightarrow p / q(\text { incl. } 1 / 0), \\
& \phi(z) \text { regular when } \operatorname{Im} z>0 .
\end{aligned}
$$

(The limit (2.22) is vertical, in the customary manner.) Now, finally, a modular form satisfying in addition the stronger relation

$$
\phi(z)=o(q z-p)^{-4} \text { as } z \rightarrow p / q(\text { incl. } 1 / 0)
$$

is called a cusp form.

MAIN THEOREM. Let $\phi(z)$ be a modular form of dimension -4 belonging to the group G. A necessary and sufficient condition that $\phi(z)$ be expressible as a linear combination of Eisenstein series $E\left(Z ; r_{t}\right)$ with real coefficients is that a third primitive function $P(z)$ exist for which

$$
\phi(z)=P^{\prime \prime \prime}(z)
$$

and for which the function $q_{T}(z)$ defined by

$$
(c z+d)^{2} P(T z)-P(z)=q_{T}(z)
$$

be a quadratic polynomial with real coefficients.

Before proceeding any further we should first note that the word "real" is the key to the theorem, since equation (2.5) leads to equation (2.21) on triple differentiation in a purely formal manner. Indeed to obtain an explicit expression for $q_{T}(z)$ all we need do is note that for a suitable point $a$ in the upper-half plane and for a suitable quadratic polynomial $Q(z)$, we can represent $P(z)$ by

$$
P(z)=\int_{a}^{z} \phi(t)(z-t)^{2} d t / 2+Q(z)
$$

and $q_{T}(z)$ by 


$$
q_{T}(z)=-\int_{a}^{T^{-1} a} \phi(t)(z-t)^{2} d t / 2+(c z+d)^{2} Q(T z)-Q(z) .
$$

We call the polynomials $q_{T}(z)$ period polynomials of $\phi(z)$ or $P(z)$. By "real period polynomial" we shall mean one with real coefficients.

The simplest illustration of a cusp form is probably $J^{\prime 2}(z) J(z)^{-4 / 3}[J(z)$ $-1]^{-1}$ corresponding to the sub-group $\varrho_{3}$ of the modular group generated as follows:

$$
G_{3}=\left\{\left(\begin{array}{ll}
1 & 3 \\
0 & 1
\end{array}\right),\left(\begin{array}{rr}
1 & 0 \\
0 & -1
\end{array}\right),\left(\begin{array}{ll}
1 & -2 \\
1 & -1
\end{array}\right),\left(\begin{array}{rr}
-1 & -2 \\
1 & 1
\end{array}\right)\right\} .
$$

Here $J(z)$ is (Klein's) invariant for the modular group. The fundamental domain of $\oint_{3}$ belongs to $J^{1 / 3}(z)$ (see [4]). It consists of three translates of the fundamental domain for the modular group extending to infinity with boundary identified by the above transformations. Thus $\oint_{3}$ has only one cusp point, one cusp form (given above), and one Eisenstein series (1.1).

3. Proof of necessity condition. Here we shall explicitly exhibit the third primitive for the Eisenstein series in (2.2). In writing an infinite series we shall from now on be asserting absolute convergence. When the summation is over an infinity of fractions $p / q$ it will be readily majorized by the corresponding sum over all pairs $(p, q)$ not necessarily prime but excluding $(0,0)$. The symbol $\sum^{\prime}$ will denote a summation omitting the possible term having $q=0$ (and majorized accordingly).

Define in accordance with the earlier paper $[1$, p. 343$]$

$$
e\left(z ; r_{t}\right)=-\frac{1}{6} \sum_{p / q \sim r_{t}}\left(z^{2}+1\right)(q+p z)(-p+q z)^{-1}\left(p^{2}+q^{2}\right)^{-2}
$$

Then

$$
\begin{aligned}
e\left(z ; r_{t}\right)= & -\frac{1}{6} \sum_{p / q \sim r_{t}}^{\prime}\left\{\frac{1}{q^{3}(q z-p)}+\frac{p\left(p^{2}+2 q^{2}\right)}{q^{3}\left(p^{2}+q^{2}\right)^{2}}\right\} \\
& +z^{2} \alpha\left(r_{t}\right)+z \beta\left(r_{t}\right)+\delta\left(r_{t}\right)\left(z^{3}+z\right) / 6
\end{aligned}
$$

where

$$
\begin{aligned}
& \alpha\left(r_{t}\right)=-\frac{1}{6} \sum_{p / q \sim r_{t}} p q^{-1}\left(p^{2}+q^{2}\right)^{-2}, \\
& \beta\left(r_{t}\right)=-\frac{1}{6} \sum_{p / q \sim r_{t}}^{\prime} q^{-2}\left(p^{2}+q^{2}\right)^{-1},
\end{aligned}
$$

and

$$
\delta\left(r_{t}\right)=\left\{\begin{array}{l}
1 \quad \text { if } r_{t} \sim 1 / 0 \\
0 \text { - otherwise }
\end{array}\right.
$$


Finally, differentiating (3.2) with respect to $z$ we find

$$
e^{\prime \prime \prime}\left(z ; r_{t}\right)=E\left(z, r_{t}\right)
$$

subject to the further conditions deducible directly from equation (3.1):

$$
e\left(i ; r_{t}\right)=0, \quad e^{\prime}\left(i ; r_{t}\right) \text { real. }
$$

For this function $e\left(z, r_{t}\right)$, we conclude

$$
z^{2} e\left(z, r_{t}\right)-e\left(z,-1 / r_{t}\right)=0
$$

directly from the definition (3.1), and by changing the summation index from $p / q$ to $p / q-1$ we find

$$
e\left(z+1, r_{t}\right)-e\left(z, r_{t}-1\right)=A_{t} z^{2}+B_{t} z+C_{t}
$$

where

$$
\begin{aligned}
A_{t}= & \alpha\left(r_{t}\right)-\alpha\left(r_{t}-1\right)+\delta\left(r_{t}\right) / 2 \\
B_{t}= & 2 \alpha\left(r_{t}\right)+\beta\left(r_{t}\right)-\beta\left(r_{t}-1\right)+\delta\left(r_{t}\right) / 2, \\
C_{t}= & -\frac{1}{6} \sum_{p / q \sim r_{t}-1}^{\prime}\left\{\frac{(p+q)\left(p^{2}+2 p q+3 q^{2}\right)}{q^{2}\left(p^{2}+2 p q+2 q^{2}\right)^{2}}-\frac{p\left(p^{2}+2 q^{2}\right)}{q^{3}\left(p^{2}+q^{2}\right)^{2}}\right\} \\
& +\alpha\left(r_{t}\right)+\beta\left(r_{t}\right)+\delta\left(r_{t}\right) / 3 .
\end{aligned}
$$

Next we observe that by a composition of the operation $z \rightarrow-1 / z$ and $z \rightarrow z+1$ we can build up any operation $T^{*}$ in the modular group (not necessarily in S). Hence by combining identities (3.5) and (3.6) we find for $T^{*} z=\left(a^{*} z\right.$ $\left.+b^{*}\right) /\left(c^{*} z+d^{*}\right)$,

$$
\left(c^{*} z+d^{*}\right)^{2} e\left(T^{*} z ; r_{t}\right)-e\left(z ; T^{*-1} r_{t}\right)=A^{*} z^{2}+B^{*} z+C^{*}
$$

for suitable real constants $A^{*}, B^{*}, C^{*}$. In particular when $T^{*}$ belongs to $\mathrm{G}$, $T^{*}=T$ and $T^{-1} r_{t}=r_{t}$. This proves the necessity portion of the main theorem.

4. Separation of the Eisenstein series. From the nature of the modular form $\phi(z)$ for every class of cusp points $p / q \sim r_{t}$ (indexed by $\left.t\right)(1 \leqq t \leqq k)$, the following limit exists and depends only on $t$ :

$$
\operatorname{Lim} \phi(z)(q z-p)^{4}=A\left(r_{t}\right) \text { as } z \rightarrow p / q \text {. }
$$

For any such point $p / q$ we consider the corresponding parabolic transformations generated by the translation

$$
Z^{\prime}=Z+h_{t}
$$

where $Z$ is a new variable given, for suitably chosen integers $r$ and $s$, by

$$
Z=S_{z}^{*}=(r z-s) /(-q z+p), \quad r p-s q=1 .
$$

(Here $S^{*}$ is in the modular group.) In terms of $z\left(=\left(S^{*}\right)^{-1} Z^{\prime}\right)$, the generating transformation is 


$$
z^{\prime}=\left\{z\left(1-h_{t} p q\right)+h_{t} p^{2}\right\} /\left\{-h_{t} q^{2} z+\left(1+h_{t} p q\right)\right\} .
$$

Now, since $\phi(z)(q z-p)^{4}=\phi\left(z^{\prime}\right)\left(q z^{\prime}-p\right)^{4}$, we have the following Fourier expansions, indexed again by $t(1 \leqq t \leqq k)$ :

$$
\phi(z)=(q z-p)^{-4}\left\{A\left(r_{t}\right)+\sum_{v=1}^{\infty} A_{\nu}\left(r_{t}\right) \exp \frac{2 \pi i v Z}{h_{t}}\right\} .
$$

Here, it must be remembered, $Z$ contains the variables $p, q$ implicitly. On triple integration, for some constants, $u(p / q), v(p / q), w(p / q)$, we obtain

$$
\begin{aligned}
P(z)= & A\left(\frac{1}{0}\right) \frac{z^{3}}{6}+\sum_{\nu=1}^{\infty} A_{\nu}\left(\frac{1}{0}\right) \cdot\left(\frac{h_{1}}{2 \pi i v}\right)^{3} \exp \frac{2 \pi i v z}{h_{1}} \\
& +w(1 / 0) z^{2}+v(1 / 0) z+u(1 / 0)
\end{aligned}
$$

where $r_{1}$ denotes the class $1 / 0$, while the other values, $p / q(\neq 1 / 0) \sim r_{t}$,

$$
\begin{aligned}
P(z)= & -\frac{A\left(r_{t}\right)}{6 q^{3}(q z-p)}+(q z-p)^{2} \sum_{v=1}^{\infty} A_{v}\left(r_{t}\right)\left(\frac{h_{t}}{2 \pi i v}\right)^{3} \exp \frac{2 \pi i v Z}{h_{t}} \\
& +u(p / q)(q z-p)^{2}+v(p / q)(q z-p)+w(p / q) .
\end{aligned}
$$

In particular, we can compute the period polynomials. Call

$$
m(z)=-h_{t} q^{2} z+\left(1+h_{t} p q\right) .
$$

Then from equation (4.4)

$$
\left(q z^{\prime}-p\right)=(q z-p) / m(z),
$$

and, in accordance with equation (2.5),

$$
m^{2}(z) P\left(z^{\prime}\right)-P(z)=U(1 / 0) z^{2}+V(1 / 0) z+W(1 / 0)
$$

where

$$
\begin{aligned}
U(1 / 0) & =A(1 / 0) h_{1} / 2, \\
V(1 / 0) & =A(1 / 0) h_{1}^{2} / 2+2 h_{1} w(1 / 0), \\
W(1 / 0) & =A(1 / 0) h_{1}^{3} / 6+w(1 / 0) h_{1}^{2}+v(1 / 0) h_{1},
\end{aligned}
$$

and for $p / q \neq 1 / 0$,

$$
m^{2} P\left(z^{\prime}\right)-P(z)=U(p / q)(q z-p)^{2}+V(p / q)(q z-p)+W(p / q)
$$

where

$$
\begin{aligned}
& U(p / q)=q^{2} h_{t}^{2} w(p / q)-q h_{t} v(p / q), \\
& V(p / q)=-A\left(r_{t}\right) h_{t}^{2} / 6-2 q h_{t} w(p / q), \\
& W(p / q)=h_{t} A\left(r_{t}\right) / 3 q .
\end{aligned}
$$


One conclusion is now immediate: If all period polynomials of equation (2.5) are real, i.e., if $U(p / q), V(p / q), W(p / q)$ are real, then the three leading terms of the expansions (4.6) and (4.7) of $P(z)$ about each cusp point have real coefficients, i.e., $A(p / q), w(p / q), v(p / q)$ are real. As a corollary to our main theorem, it would eventually follow that the $u(p / q)$ are then real for a proper choice of additive constant in $P(z)$.

We can simplify the sufficiency portion of the main theorem by subtracting from our $\phi(z)$ (with real period polynomials) the linear combination $A\left(r_{t}\right) E\left(z ; r_{t}\right)$. The difference, which we may also denote by $\phi(z)$, would then be a cusp form. Thus it suffices to show that a cusp form with real period polynomiuls is identically zero.

5. Formal role of perturbation theory. To see the relevance of period polynomials to perturbation theory, let us assume that $P(z)$ has real period polynomials. Then first of all define a quadratic polynomial with real coefficients

$$
q_{0}(z)=\alpha z^{2}+\beta z+\gamma
$$

such that $P_{0}(z)=P(z)+q_{0}(z)$ satisfies the condition

$$
\begin{gathered}
P_{0}\left(z_{0}\right)=0, \\
d P_{0}\left(z_{0}\right) / d z \text { real }
\end{gathered}
$$

for some $z_{0}$ with $\operatorname{Im} z_{0}>0$. We find $\alpha, \beta, \gamma$ from the linear equations

$$
\begin{aligned}
P\left(z_{0}\right)+\alpha z_{0}^{2}+\beta z_{0}+\gamma & =0, \\
\overline{P\left(z_{0}\right)}+\alpha \overline{z_{0}}+\beta \overline{z_{0}}+\gamma & =0, \\
P^{\prime}\left(z_{0}\right)+2 \alpha z_{0}+\beta & =\overline{P^{\prime}\left(z_{0}\right)}+2 \alpha \overline{z_{0}}+\beta
\end{aligned}
$$

with determinant $2\left(z_{0}-\overline{z_{0}}\right)^{2} \neq 0$.

Now $P_{0}(z)$ still has real period polynomials. With it we define for a real small parameter $\epsilon$

$$
\zeta_{\epsilon}(z)=z+\epsilon P_{0}(z)
$$

an infinitesimal mapping function. We can define for it an infinitesimal variation of $T$, any matrix of $\mathcal{G}$ (see equation 2.1).

$$
T_{\epsilon}=\left(\begin{array}{ll}
a+A \epsilon & b+B \epsilon \\
c+C \epsilon & d+D \epsilon
\end{array}\right)
$$

such that

$$
\zeta_{\epsilon}(T z)=T_{\epsilon}\left(\zeta_{\mathrm{e}}(z)\right)+o(\epsilon) .
$$

In fact to determine $T_{\mathrm{c}}$ we write down the (real) period polynomial (see (2.5)), 


$$
(c z+d)^{2} P_{0}(T z)-P_{0}(z)=A_{T} z^{2}+B_{T} z+C_{T} .
$$

Then differentiating equation (5.7) with respect to $\epsilon$, at $\epsilon=0$, we find

$$
\begin{aligned}
A c-C a & =A_{T}, \\
A d+B c-C b-D a & =B_{T}, \\
B d-D b & =C_{T},
\end{aligned}
$$

and from the unimodular property of $T_{\epsilon}$ (to within $\epsilon$ ),

$$
d A-c B-b C+a D=0 .
$$

Equations (5.91) and (5.92) in $A, B, C, D$ have determinant $-(a d-b c)^{2}=-1$, which fixes real values $A, B, C, D$ and hence a real $T_{\text {c. }}$.

Thus we can think of $\zeta_{\epsilon}(z)$ as a function defining an infinitesimal perturbtion mapping of the upper half $z$-plane onto itself, first of all, leaving point and direction invariant at $z=z_{0}$ and, secondly, leaving the upper half plane invariant, to the extent that the Fuchsian group has a real perturbation. The remainder of the paper amounts, heuristically speaking, to showing that the boundary variations induced by $\zeta_{\mathrm{e}}(z)$ are exactly the variations at the cusp points, and that such variations produce just Eisenstein series.

In the earlier paper on the subject [1], a type of variation was used for which $\zeta_{\epsilon}(z)$ could only be approximated, while from geometrical considerations $T_{c}$ was known exactly (with no error term in equation (5.7)). In the present work our knowledge will be less explicit but the fact that our variations preserve the boundary $(\operatorname{Im} z=0)$ of the upper half plane will still be the characterizing feature of the Eisenstein series.

6. Perturbation interpretation of real period polynomials. To see the geometric significance of real coefficients we first reduce the problem by the assumption (see $\$ 4$ ) that all $A\left(r_{t}\right)=0$ or that the modular form $\phi$ is a cusp form. Then under the assumption that the period polynomials of some $\phi(z)$ are real, we subtract $w(1 / 0) z^{2}+v(1 / 0) z+\operatorname{Re} u(1 / 0)$ from $P(z)$ in accordance with formula (4.6) leaving $P_{\infty}(z)$ with the property

$$
P_{\infty}\left(z+h_{1}\right)=P_{\infty}(z)
$$

and then, for some pure-imaginary constant $c_{\infty}$,

$$
P_{\infty}(z)=\int_{\infty}^{z} \phi(t)(z-t)^{2} d t / 2+c_{\infty},
$$

since, according to formula (4.6),

$$
P_{\infty}(z) \rightarrow c_{\infty} \text { as } \operatorname{Im} z \rightarrow \infty .
$$

Our sufficiency proof finally reduces to showing that under the assumption of real period polynomials, $P_{\infty}(z) \equiv c_{\infty}$, with the incidental result that $c_{\infty}=0$.

We define the coefficients $w(p / q), v(p / q)$, etc., with regard to $P_{\infty}(z)$ as in 
$\S 4$. We parametrize $\mathfrak{C}^{*}$ a circle of fixed radius $\rho$ tangent to the real axis, from above, at a fixed $z=p / q(\neq 1 / 0)$, as follows:

$$
z-p / q=2 \rho \sin \theta e^{i \theta}, \quad 0<\theta<2 \pi .
$$

It is easily seen that according to formula (4.7)

$$
\exp 2 \pi i Z / h_{t}=\exp -2 \pi /\left(2 \rho q^{2} h_{t}\right),
$$

so that formula (4.7) always applied to $P_{\infty}(z)$ has the form

$$
P_{\infty}(z)=w(p / q)+v(p / q)(q z-p)+O(1)(q z-p)^{2} .
$$

We finally obtain, permitting $w(p / q), v(p / q)$ to be nonreal for the moment,

$$
\begin{aligned}
\operatorname{Im} P_{\infty}(z)= & \operatorname{Im} w(p / q)+\operatorname{Re} v(p / q) 2 \rho q \sin ^{2} \vartheta \\
& +\operatorname{Im} v(p / q) 2 \rho q \sin \vartheta \cos \vartheta+4 \rho^{2} q^{2} \sin ^{2} \vartheta O(1)
\end{aligned}
$$

which we shall compare with

$$
\operatorname{Im} z=2 \rho \sin ^{2} \theta, \quad \text { for } 0<\theta<2 \pi .
$$

Hence we are led to the following variational criterion of real coefficients: The functions $\zeta(z)=z \pm \epsilon P_{\infty}(z) m a p \mathfrak{e}^{*}$ into the upper half $\zeta$-plane again, i.e.,

$$
\epsilon\left|\operatorname{Im} P_{\infty}(z)\right|<\operatorname{Im} z
$$

for $\epsilon$ a sufficiently small positive quantity, if and only if, the period polynomials have real coefficients, i.e., if and only if $w(p / q), v(p / q)$ are real.

7. Estimates for the sufficiency proof. The sufficiency proof will be consummated by a return to equations (6.7) and (6.8) with estimates so improved that from now on the $O$ symbol will refer to $p / q$ as well as $\theta$ (when relevant). To avoid unnecessary difficulties in notation assume the fundamental domain for group $\mathrm{G}$ has only one cusp-point.

Our assumption is now that a given cusp form $\phi(z)$ leads to a third primitive $P_{\infty}(z)$ (see equation (6.2)), with real values for $v(p / q)$ and $\left.w(p / q)\right)$. We shall show $\phi(z) \equiv 0$.

First of all, note that for a cusp form $v(p / q), w(p / q)$ are expressible in terms of derivatives of $P_{\infty}(z)$ since

$$
\begin{aligned}
P_{\infty}(z)= & P_{\infty}(p / q)+P_{\infty}^{\prime}(p / q)(z-p / q) \\
& +P_{\infty}^{\prime \prime}(p / q)(z-p / q)^{2} / 2+(q z-p)^{2}\left[P_{\infty}(Z)-c_{\infty}\right]
\end{aligned}
$$

according to equation (4.5). Next we observe that in the expansion

$$
\phi(z)=\sum_{\nu=1}^{\infty} A_{\nu} \exp 2 \pi i \nu z / h \quad\left(h_{1}=h\right),
$$

according to a theorem of Kloosterman [3], 


$$
A_{\nu}=o\left(\nu^{1+\mu}\right)
$$

for any constant $\mu$ satisfying

$$
\mu>7 / 8 \text {. }
$$

From this we form the estimates

$$
\begin{aligned}
q v(p / q) & =P_{\infty}^{\prime}(p / q)=o\left(q^{\mu}\right), \\
2 q^{2} u(p / q) & =P_{\infty}^{\prime \prime}(p / q)=O\left(q^{2}\right) .
\end{aligned}
$$

To see estimate (7.51) we write (from formula (6.2))

$$
\begin{aligned}
P^{\prime}(p / q) & =-\int_{\infty}^{p / q} \phi(t)(t-p / q) d t=-\int_{\infty}^{p / q+i / q}-\int_{p / q+i / q}^{p / q} \\
& =-\int_{\infty}^{p / q+i / q} \phi(t)(t-p / q) d t-\int_{\infty}^{-r / q+i / q} \phi(z)(r / q+z) d z
\end{aligned}
$$

where $t=(p z+s) /(q z+r)$, according to a transformation of $\mathcal{G}$, and $\phi(t)$ is transformed, in the second integral according to property (2.21). Thus by substitution of the Fourier series (7.2), we find

$$
\begin{aligned}
P^{\prime}(p / q)=\sum_{\nu=1}^{\infty} A_{\nu} & \left(\exp -\frac{2 \pi \nu}{q h}\right)\left(\frac{h}{2 \pi \nu q}-\frac{h^{2}}{4 \pi^{2} \nu^{2}}\right) \\
& \left(\exp \frac{2 \pi i \nu p}{h q}-\exp \frac{2 \pi i \nu r}{h q}\right) .
\end{aligned}
$$

The estimate (7.51) (and similarly (7.52)), follow now from the easy result

$$
\sum_{\nu=1}^{\infty} \nu^{\sigma} \exp -\frac{2 \pi \nu}{h q}=\left\{\begin{array}{lr}
O\left(q^{1+o}\right), & \text { if } g>-1, \\
O(\log q), & g=-1, \\
O(1), & g<-1 .
\end{array}\right.
$$

8. Completion of sufficiency proof. We next consider the system of discs (solid circles) given by the inequalities in the $z$-plane

$$
\operatorname{Im} Z>\lambda
$$

where $Z=S^{*} z$ is the most general transformation of the complete modular group. The discs are bounded by circles in the upper half-plane tangent to the real axis at $p / q(\neq 1 / 0)$ with radius

$$
\rho=1 /\left(2 \lambda q^{2}\right)
$$

(in addition to the "infinite disc" $\operatorname{Im} z>\lambda$ ). Under the simplifying assumption that the group $\subseteq$ has one cusp point, all such discs are equivalent under mo- 
tions of $\mathrm{g}$. As was known to Hermite, these discs completely cover the upper half-plane if $\lambda$ is a constant satisfying

$$
\lambda<3^{1 / 2} / 2 \text {. }
$$

Now using the value of $\rho$ in equation (8.2) we obtain from equation (6.7) the estimate

$$
\operatorname{Im} P_{\infty}(z)=o\left(q^{\mu}\right) \sin ^{2} \theta / q^{2}
$$

where $z$ is associated with the disc at $p / q$. By differentiation of equation (7.1), we similarly obtain

$$
P_{\infty}^{\prime}(z)=o\left(q^{\mu}\right) .
$$

We now consider the Farey subdivision of the real axis of order $N$, consisting of all positive and negative reduced fractions $p / q$ with $0<q \leqq N$. We call $R^{*}(N)$ the portion of the upper half-plane covered by the infinite disc and all discs belonging to the fractions $p / q$ in the Farey subdivision. The highest boundary point of $\Omega^{*}(N)$ has its imaginary part at most equal to $2 / N^{2}$. This follows from the fact that in the Ford configuration (with $\lambda=1$ ) (see [6]), any two Farey neighbors $p_{1} / q_{1}, p_{2} / q_{2}$ have tangent circles with point of tangency at distance from the real axis $=\left(q_{1}^{2}+q_{2}^{2}\right)^{-1} \leqq 2\left(q_{1}+q_{2}\right)^{-2}$ $\leqq 2 / N^{2}$. Thus from equations (8.41) and (6.4) we conclude, taking $N=\epsilon^{-1 / \mu}$, that for $\epsilon$ a small positive quantity, the function

$$
\zeta(z)=z+\epsilon P_{\infty}(z)
$$

maps $R^{*}\left(\epsilon^{-1 / \mu}\right)$ into a region (say) $R_{0}^{*}\left(\epsilon^{-1 / \mu}\right)$ of the $\zeta$-plane. The mapping is unique because, by virtue of the estimate (8.42), $\left|\epsilon P_{\infty}^{\prime}(z)\right| \rightarrow 0$ for $z$ in $R^{*}\left(\epsilon^{-1 / \mu}\right)$ as $\epsilon \rightarrow 0$.

Perform the transformation

$$
\begin{aligned}
w & =\exp 2 \pi i z / h, \\
w^{\prime} & =\exp 2 \pi i \zeta / h ;
\end{aligned}
$$

then the mapping (8.5) determines a mapping of $R\left(\epsilon^{-1 / \mu}\right)$, the $w$-image of $R^{*}\left(\epsilon^{-1 / \mu}\right)$, into $\Omega_{0}\left(\epsilon^{-1 / \mu}\right)$, the $w^{\prime}$-image of $R_{0}^{*}\left(\epsilon^{-1 / \mu}\right)$. Specifically if we set

$$
P_{\infty}(z)=G(w)
$$

we find that the transformation (8.5) becomes

$$
w^{\prime}=w \exp 2 \pi i \epsilon G(w) / h,
$$

a mapping which preserves the origin and the direction at the origin (since $c_{\infty}$ is pure-imaginary). But by our earlier estimates the boundaries of $R\left(\epsilon^{-1 / \mu}\right)$ and $R_{0}\left(\epsilon^{-1 / \mu}\right)$ differ from the unit circle (and from one another) by a perturbation of size $O\left(2 / N^{2}\right)=O\left(\epsilon^{2 / \mu}\right)=o(\epsilon)$ for $\mu<2$, while the resultant in- 
terior mapping (8.8) is actually a perturbation of order $\epsilon$ unless $G(w)=0$. This completes the sufficiency proof of the main problem.

9. Concluding remarks. The first thing we might notice is that Kloosterman's estimation (7.3) of the Fourier coefficients is much too sharp. A closer examination of the steps shows that it would suffice to know that $A_{\nu}=O\left(\nu^{3}\right)$, which applies to the Eisenstein series as well as the cusp forms.

One might infer that a purely function-theoretic proof of the main theorem should be possible employing no such number-theoretic device as the Farey subdivision, perhaps along the lines of $\$ 5$.

We should add that $\$ 5$ is more than a heuristic motivation for the use of $z+\epsilon P(z)$. If we reverse the sequence of equations, from (5.7) to (5.5), our main theorem gives a proof that cusp forms are not definable by a variational process which forms a real perturbation of the transformation matrices of the group $\mathcal{S}$ (since such a perturbation leads to a real period polynomial). In fact, in $\$ 6$ we saw that variations arising from cusp forms would cause some of the "discs" to "perturb" the real axis by moving below the upper halfplane. The earlier paper [1] indicates how Eisenstein series, on the other hand, could be constructed by a variational procedure which forms a real perturbation transformation of the group $\mathcal{G}$.

\section{REFERENCES}

1. H. Cohn, Modular functions defined by perturbation mappings, Lectures on Functions of a Complex Variable, Ann Arbor, 1955, pp. 341-348.

2. M. Eichler, Quadratische Formen und Orthogonale Gruppen, Berlin, 1952, pp. 150-152.

3. T. Estermann, Vereinfachter Beweis eines Satzes von Kloosterman, Abh. Math. Sem. Hansischen Univ. vol. 7 (1930) pp. 82-98.

4. R. Fricke and F. Klein, Vorlesungen über die Theorie der elliptischen Modulfunktionen, Leipzig, 1890, pp. 623 ff.

5. H. Petersson, Konstruktion der Modulformen von positiver reeler Dimension und die vollständige Bestimmung ihrer Fourierkoeffizienten, Heidelberg Akad. Wiss. Sitzungsber. (1950) pp. 67-77.

6. H. Rademacher, On the expansion of the partition function in a series, Ann. of Math. vol. 44 (1943) pp. 416-422.

7. M. Schiffer and D. C. Spencer, Functionals of finite Riemann surfaces, Princeton, 1954.

WAYNE UNIVERSITY,

Detroit, Mich.

STANFORD UNIVERSITY,

Stanford, Calif. 